\title{
DP1 AND COMPLETELY CONTINUOUS OPERATORS
}

\author{
ELIZABETH M. BATOR and DAWN R. SLAVENS
}

\author{
Received 11 February 2003
}

\begin{abstract}
W. Freedman introduced an alternate to the Dunford-Pettis property, called the DP1 property, in 1997. He showed that for $1 \leq p<\infty,\left(\bigoplus_{\alpha \in \mathscr{A}} X_{\alpha}\right)_{p}$ has the DP1 property if and only if each $X_{\alpha}$ does. This is not the case for $\left(\bigoplus_{\alpha \in \mathscr{A}} X_{\alpha}\right)_{\infty}$. In fact, we show that $\left(\bigoplus_{\alpha \in \mathscr{A}} X_{\alpha}\right)_{\infty}$ has the DP1 property if and only if it has the DunfordPettis property. A similar result also holds for vector-valued continuous function spaces.
\end{abstract}

2000 Mathematics Subject Classification: 46B20, 46B28, 46G10, $28 \mathrm{~B} 05$.

Let $X$ and $Y$ be Banach spaces and $T: X \rightarrow Y$ a bounded linear operator. We say that $T$ is a DP1 operator if whenever $\left(x_{n}\right)$ is a sequence of norm one elements in $X$ such that $\left(x_{n}\right) \rightarrow x \in X$ weakly with $\|x\|=1$, then $\left(T\left(x_{n}\right)\right) \rightarrow$ $T(x)$ in norm. A Banach space $X$ is then said to have the DP1 property if, for each Banach space $Y$, a bounded linear operator $T: X \rightarrow Y$ must be DP1 whenever it is weakly compact. Freedman introduced this property in [5], and referred to it as a compromise between the Dunford-Pettis property (DPP) and the Kadec-Klee property (KKP). (Recall that a Banach space $X$ has the DPP if every weakly compact $T: X \rightarrow Y$ is completely continuous, that is, $T$ sends weakly null sequences to norm null sequences. The Banach space $X$ has KKP if whenever $\left(x_{n}\right)$ is a sequence in $X$ such that $\left(x_{n}\right) \rightarrow x$ weakly and each $x_{n}$ and $X$ belong to the unit sphere of $X, S_{X}$, then $\left\|x_{n}-x\right\| \rightarrow 0$.) He also showed that, although the DP1 property is preserved by linear isometries, it is not preserved by isomorphisms. It then follows that the collection of DP1 operators does not form an operator ideal. Thus the relationship between DP1 operators with better known classes of operators is somewhat unpredictable.

Freedman proved that if $X=\left(\bigoplus_{\alpha \in \mathscr{A}} X_{\alpha}\right)_{p}, 1 \leq p<\infty$, then $X$ has the DP1 property or KKP if and only if each $X_{\alpha}$ has the DP1 property or KKP, respectively. This is certainly not the case for $X=\left(\bigoplus_{\alpha \in \mathscr{A}} X_{\alpha}\right)_{\infty}$, as the following theorem and corollary indicate. (We will assume that $\mathscr{A}$ has at least two elements, and each $X_{\alpha}$ is nontrivial.) We first define the following terms.

If $\left\{X_{\alpha}: \alpha \in \mathscr{A}\right\}$ is a family of Banach spaces and $1 \leq p<\infty$, define

$$
\left(\bigoplus_{\alpha \in \mathscr{A}} X_{\alpha}\right)_{p}=\left\{x=\left(x_{\alpha}\right) \in \prod_{\alpha \in \mathscr{A}} X_{\alpha}: \sum_{\alpha \in \mathscr{A}}\left\|x_{\alpha}\right\|^{p}<\infty\right\},
$$


with $\|x\|=\left(\sum_{\alpha}\left\|x_{\alpha}\right\|^{p}\right)^{1 / p}$, and

$$
\left(\bigoplus_{\alpha \in \mathscr{A}} X_{\alpha}\right)_{\infty}=\left\{x=\left(x_{\alpha}\right) \in \prod_{\alpha \in \mathscr{A}} X_{\alpha}: \sup _{\alpha}\left\|x_{\alpha}\right\|<\infty\right\},
$$

with $\|x\|=\sup _{\alpha}\left\|x_{\alpha}\right\|$.

THEOREM 1. Let $X=\left(\bigoplus_{\alpha \in \mathscr{A}} X_{\alpha}\right)_{\infty}$. If $Z$ is a Banach space, then a bounded linear operator $T: X \rightarrow Z$ is DP1 if and only if it is completely continuous.

Proof. Suppose that $T: X \rightarrow Z$ is DP1 but not completely continuous. Let $\beta \in \mathscr{A}, Y_{1}=X_{\beta}$, and $Y_{2}=\left(\bigoplus_{\alpha \neq \beta \in \mathscr{A}} X_{\alpha}\right)$. Thus $X=Y_{1} \oplus_{\infty} Y_{2}$. Since $T$ is not completely continuous, there exists a normalized weakly null sequence $\left(x_{n}, y_{n}\right)$ in $Y_{1} \oplus_{\infty} Y_{2}$ and $\epsilon>0$ such that $\left\|T\left(\left(x_{n}, y_{n}\right)\right)\right\|>\epsilon$ for each $n \in \mathbb{N}$. Define $T_{1}: Y_{1} \rightarrow Z$ and $T_{2}: Y_{2} \rightarrow Z$ by $T_{1}(x)=T((x, 0))$ and $T_{2}(y)=T((0, y))$. It is clear that $T((x, y))=T_{1}(x)+T_{2}(y)$. Thus, by passing to a subsequence if needed, we may assume that either (a) $\left\|T_{1}\left(x_{n}\right)\right\|>\epsilon / 2$ or (b) $\left\|T_{2}\left(y_{n}\right)\right\|>\epsilon / 2$, for each $n \in \mathbb{N}$. Without loss of generality, we assume that (a) holds. Let $y \in Y_{2}$ such that $\|y\|=1$. As $\left(x_{n}\right)$ is weakly null in $X_{1}$, the sequence $\left(\left(x_{n}, y\right)\right)_{n=1}^{\infty}$ converges weakly to $(0, y)$, and each $\left(x_{n}, y\right)$ and $(0, y)$ belong to $S_{X_{1} \oplus X_{2}}$. As $T$ is $\mathrm{DP} 1, T\left(\left(x_{n}, y\right)\right) \rightarrow T((0, y))$ in norm. Therefore, $T_{1}\left(x_{n}\right) \rightarrow 0$ in norm, which is a contradiction.

COROLlary 2. Let $X=\left(\bigoplus_{\alpha \in \mathscr{A}} X_{\alpha}\right)_{\infty}$. Then $X$ has the DP1 property if and only if it has the DPP. Likewise, $X$ has the KKP if and only if $X$ is a Schur space.

Observe that if $\left(\bigoplus_{\alpha \in \mathscr{A}} X_{\alpha}\right)_{\infty}$ has the DP1 property (KKP) and hence the DPP (Schur property), then each $X_{\alpha}$ has the DPP (Schur property). If $\mathscr{A}$ is finite, then the converse also holds. However, the converse need not be true for infinite $A$ A. For instance, if $X=\left(\bigoplus_{n \in \mathbb{N}} \ell_{2}^{n}\right)_{\infty}$, then $X$ contains a complemented copy of $\ell_{2}$ (see [6, page 61]) and hence $X$ does not have the DPP (Schur property), even though each $\ell_{2}^{n}$ has the DPP (Schur property).

We will now consider DP1 operators from $C(H, X)$ to $Y$, where $X$ and $Y$ are Banach spaces, $H$ is a compact Hausdorff space with Borel subsets $\Sigma$, and $C(H, X)$ is the Banach space (sup-norm) of $X$-valued continuous functions on $H$. If $T: C(H, X) \rightarrow Y$ is a bounded linear map, then there exists a unique weakly regular set function $m: \Sigma \rightarrow L\left(X, Y^{* *}\right)$ such that $\tilde{m}(H)<\infty$ and $T(f)=\int f d m$ for each $f \in C(H, X)$. This is denoted by $m \leftrightarrow T$. (Note that $\tilde{m}(A)=\sup _{\pi \in \Pi(A)}\left\{\left\|\sum_{A_{i} \in \pi} m\left(A_{i}\right) x_{i}\right\|:\left\|x_{i}\right\| \leq 1\right\}$, where $\Pi(A)$ denotes the collection of finite $\Sigma$ partions of $A$. See [2] or [4, Chapter 3] for a discussion of the Riesz representation theorem in this setting.) The operator $m \leftrightarrow T$ is said to be strongly bounded if $\tilde{m}\left(A_{i}\right) \rightarrow 0$ for each pairwise disjoint sequence $\left(A_{i}\right)$ of members of $\Sigma$.

LEMMA 3. If $m \leftrightarrow T: C(H, X) \rightarrow Y$ is a DP1 operator, then $m$ is strongly bounded. 
Proof. Suppose that $m \leftrightarrow T: C(H, X) \rightarrow Y$ is a DP1 operator, but $m$ is not strongly bounded. Then there exist sequences $\left(U_{n}\right)$ of pairwise disjoint open subsets of $H$ and $\left(f_{n}\right)$ in $C(H, X)$ such that $\left\|f_{n}\right\|=1$, $\operatorname{supp}\left(f_{n}\right) \subseteq U_{n}$, and $\left\|T\left(f_{n}\right)\right\|>\epsilon$ for each $n \in \mathbb{N}$ (see [1, Theorem 2.8]). Define $g_{n}=f_{1}+f_{n+1}$ for each $n$. As $\left(f_{n}\right)$ is weakly null, $\left(g_{n}\right) \rightarrow f_{1}$ weakly and $\left\|g_{n}\right\|=1$ for each $n \in \mathbb{N}$. Since $T$ is DP1, $T\left(g_{n}\right) \rightarrow T\left(f_{1}\right)$ in norm, and hence $T\left(f_{n+1}\right) \rightarrow 0$ in norm. This is a contradiction.

THEOREM 4. Let $X$ and $Y$ be Banach spaces, $H$ a compact Hausdorff space containing at least two elements, and $\Sigma$ the Borel subsets of $H$. Then a bounded linear operator $m \leftrightarrow T: C(H, X) \rightarrow Y$ is DP1 if and only if it is completely continuous. Thus $C(H, X)$ has the DP1 property if and only if it has the DPP.

Proof. Suppose that $m \leftrightarrow T: C(H, X) \rightarrow Y$ is DP1 but not completely continuous. Then there exists a normalized weakly null sequence $\left(f_{n}\right)$ in $C(H, X)$ and $\epsilon>0$ such that $\left\|T\left(f_{n}\right)\right\|>6 \epsilon$. Let $U$ and $V$ be nonempty open subsets of $H$ such that $\bar{U} \cap \bar{V}=\varnothing$. As $T$ is DP1, Lemma 3 tells us that $m$ is strongly bounded, and hence we may choose a regular nonnegative measure $\mu$ defined on $\sum$ such that $\lim _{\mu(A) \rightarrow 0} \tilde{m}(A)=0$.

For each $n \in \mathbb{N}$, either (a) $\left\|\int_{\bar{U}} f_{n} d m\right\|>3 \epsilon$ or (b) $\left\|\int_{H \backslash \bar{U}} f_{n} d m\right\|>3 \epsilon$. By passing to a subsequence, we will assume that (a) holds for all $n \in \mathbb{N}$. Let $\varphi_{1}$ : $H \rightarrow[0,1]$ be a continuous function such that $\varphi(\bar{U})=1$ and $\varphi(\bar{V})=0$. Next let $\delta>0$ be such that if $\mu(A)<\delta$, then $\tilde{m}(A)<\epsilon$. Use Lusin's theorem to obtain a continuous function $\varphi_{2}: H \rightarrow[0,1]$ such that if $A=\left\{x: \varphi_{2}(x) \neq \chi_{\bar{U}}(x)\right\}$, then $\mu(A)<\delta$. Let $g \in C(H, X)$ such that $\|g\|=1$ and the support of $g$ is contained in $\bar{V}$. For each $n \in \mathbb{N}$, define $g_{n}=\varphi_{1} \varphi_{2} f_{n}$. This sequence is weakly null in $C(H, X)$ as $\left(f_{n}\right)$ is. Thus $\left(g_{n}+g\right)$ converges weakly to $g$. Also $\left\|g_{n}+g\right\|=1$ for each $n \in \mathbb{N}$. As $T$ is DP1, we have that $\left\|T\left(g_{n}+g\right)-T(g)\right\| \rightarrow 0$, and thus $\left\|T\left(g_{n}\right)\right\| \rightarrow 0$. But

$$
\begin{aligned}
\left\|T\left(g_{n}\right)\right\| & =\left\|\int_{\bar{U} \cup A} g_{n} d m\right\| \\
& \geq\left\|\int_{\bar{U} \backslash A} g_{n} d m\right\|-\left\|\int_{A} g_{n} d m\right\| \\
& \geq\left\|\int_{\bar{U} \backslash A} f_{n} d m\right\|-\tilde{m}(A)\left\|g_{n}\right\| \\
& \geq\left\|\int_{\bar{U}} f_{n} d m\right\|-\tilde{m}(A)\left(\left\|g_{n}\right\|+\left\|f_{n}\right\|\right) \\
& >3 \epsilon-2 \epsilon=\epsilon,
\end{aligned}
$$

which yields the desired contradiction.

Now suppose that (b) holds. Use the fact that $\mu$ is a regular "control" measure for $\tilde{m}$ to obtain a closed subset $K \subseteq H \backslash \bar{U}$ such that $\left\|\int_{K} f d m\right\|>3 \epsilon$. The proof follows as above by replacing, in the proof of case (a), $\bar{U}$ and $\bar{V}$ with $K$ and $\bar{U}$, 
respectively, and again obtaining the desired contradiction. Hence $T$ must be completely continuous.

We conclude with the following observation. Since every DP1 operator on $\left(\bigoplus_{\alpha \in \mathscr{A}} X_{\alpha}\right)_{\infty}$ and $C(H, E)$ are completely continuous, they are also unconditionally converging. Perhaps a reasonable question may be whether or not all DP1 operators are unconditionally converging. (Of course it is certainly the case that there are unconditionally converging operators that are not DP1.) To consider the converse notion, let $\||\cdot|\|$ denote the norm on $c_{o}$, equivalent to the usual norm, defined by Day in [3]. It was shown in [7] that $\left(c_{o},\||\cdot|\|\right)$ is locally uniformly rotund, and hence has KKP. (See [8] for more details about $\left(c_{o},\||\cdot|\|\right)$.) It is straightforward to show that $X$ has the KKP if and only if for each $Y$, every bounded linear operator $T: X \rightarrow Y$ (in particular, any isomorphism of $X$ ) is DP1. Thus, using a classical result of Besagga and Pelczynski, we obtain the following theorem.

THEOREM 5. If $X$ and $Y$ are Banach spaces, then a DP1 operator $T: X \rightarrow Y$ is not unconditionally converging if and only if $X$ contains a closed subspace $Z$ such that $Z$ is isometrically isomorphic to $\left(c_{o},\||\cdot|\|\right)$ and $\left.T\right|_{Z}$ is an isomorphism.

\section{REFERENCES}

[1] C. A. Abbott, E. M. Bator, R. G. Bilyeu, and P. W. Lewis, Weak precompactness, strong boundedness, and weak complete continuity, Math. Proc. Cambridge Philos. Soc. 108 (1990), no. 2, 325-335.

[2] J. K. Brooks and P. W. Lewis, Linear operators and vector measures, Trans. Amer. Math. Soc. 192 (1974), 139-162.

[3] M. M. Day, Strict convexity and smoothness of normed spaces, Trans. Amer. Math. Soc. 78 (1955), 516-528.

[4] N. Dinculeanu, Vector Measures, International Series of Monographs in Pure and Applied Mathematics, vol. 95, Pergamon Press, New York, 1967.

[5] W. Freedman, An alternative Dunford-Pettis property, Studia Math. 125 (1997), no. 2, 143-159.

[6] W. B. Johnson and J. Lindenstrauss (eds.), Handbook of the Geometry of Banach Spaces. Vol. I, North-Holland Publishing, Amsterdam, 2001.

[7] J. Rainwater, Local uniform convexity of Day's norm on $c_{0}(\Gamma)$, Proc. Amer. Math. Soc. 22 (1969), 335-339.

[8] M. A. Smith, Some examples concerning rotundity in Banach spaces, Math. Ann. 233 (1978), no. 2, 155-161.

Elizabeth M. Bator: Department of Mathematics, University of North Texas, P.O. Box 311400 Denton, TX 76203-1400, USA

E-mail address: bator@unt.edu

Dawn R. Slavens: Department of Mathematics, Midwestern State University, 3410 Taft Blvd, Wichita Falls, TX 76308, USA

E-mail address: dawn.s1avens@mwsu .edu 


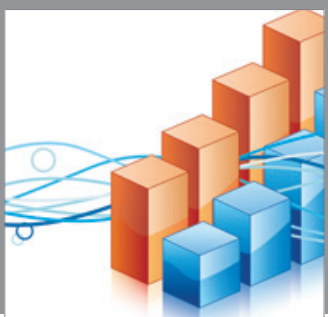

Advances in

Operations Research

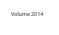

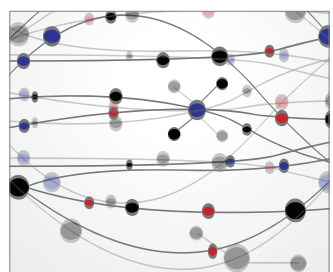

\section{The Scientific} World Journal
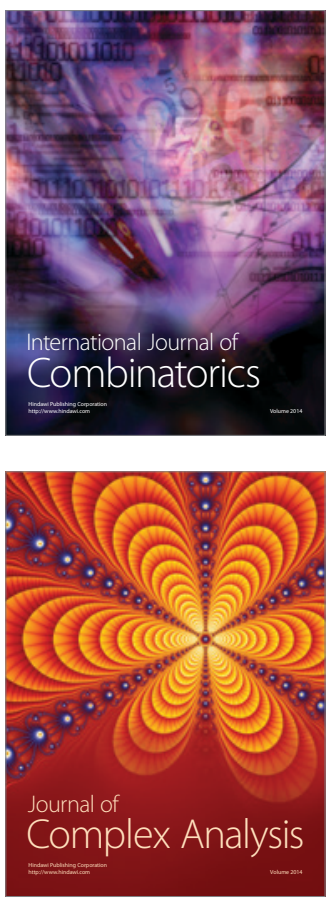

International Journal of

Mathematics and

Mathematical

Sciences
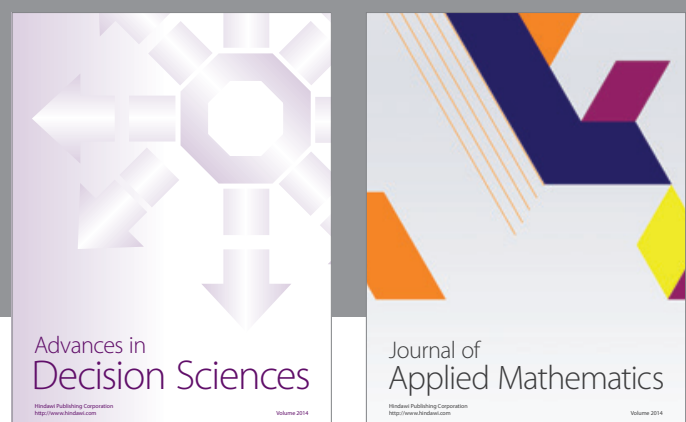

Journal of

Applied Mathematics
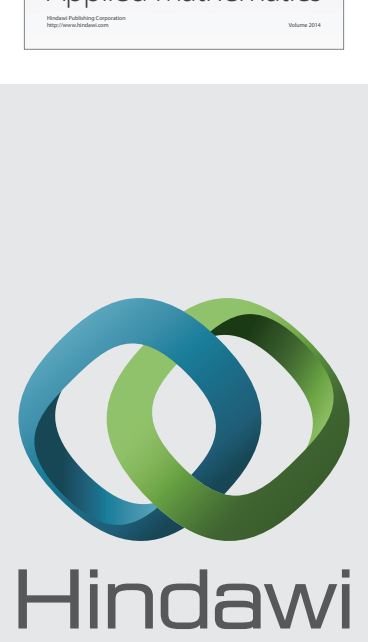

Submit your manuscripts at http://www.hindawi.com
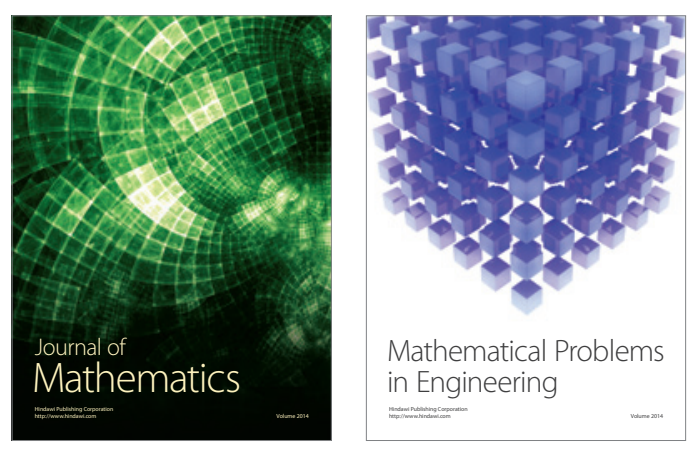

Mathematical Problems in Engineering
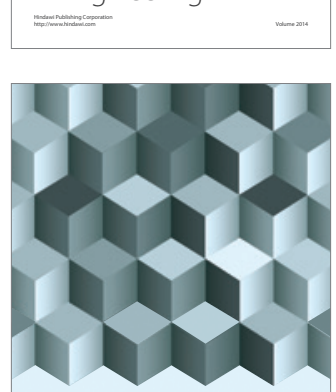

Journal of

Function Spaces
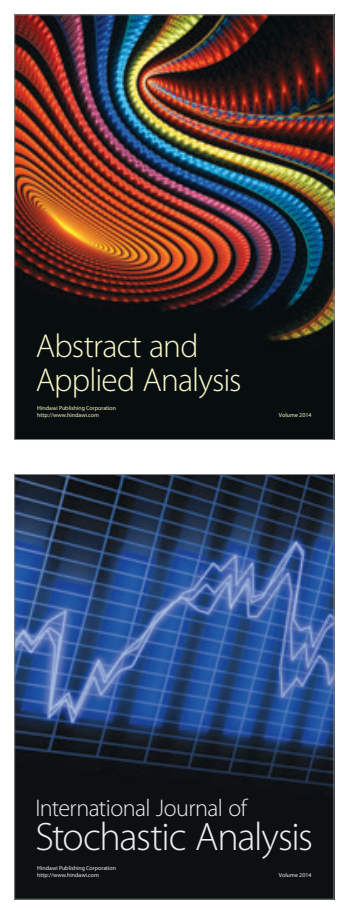

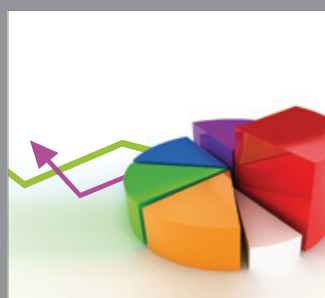

ournal of

Probability and Statistics

Promensencen
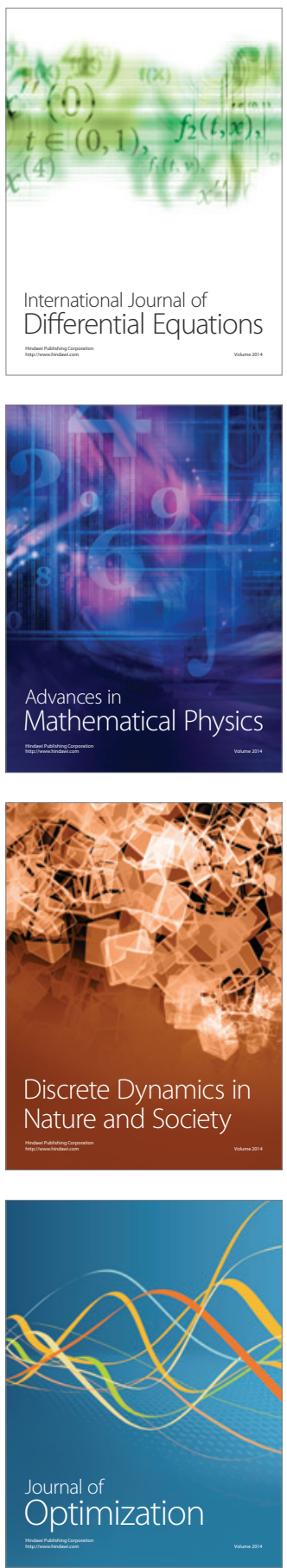